\title{
Generalized Dupin Cyclides with Rational Lines of Curvature
}

\author{
Martin Peternell \\ Institute of Discrete Mathematics and Geometry, \\ Vienna University of Technology, \\ Wiedner Hauptstrasse 8-10, 1040 Wien, Austria \\ http://www.dmg.tuwien.ac.at
}

\begin{abstract}
Dupin cyclides are algebraic surfaces of order three and four whose lines of curvature are circles. These surfaces have a variety of interesting properties and are aesthetic from a geometric and algebraic viewpoint. Besides their special property with respect to lines of curvature they appear as envelopes of one-parameter families of spheres in a twofold way. In the present article we study two families of canal surfaces with rational lines of curvature and rational principal curvatures, which contain the Dupin cyclides of order three and four as special instances in each family. The surfaces are constructed as anticaustics with respect to parallel illumination and reflection at tangent planes of curves on a cylinder of rotation.
\end{abstract}

Keywords: rational lines of curvature, canal surface, envelope of spheres, anticaustic by reflection

\section{Introduction}

Dupin cyclides are among the famous surfaces studied in classical geometry and date back to the nineteenth century, see $[4,6]$. These surfaces are characterized by the fact that their lines of curvature are circles. These two families of circles lie in two pencils of planes and the tangent planes along a fixed circle envelope a cone of revolution. Dupin cyclides are special instances of the larger class of Darboux cyclides which denote algebraic surfaces of order four having the ideal conic $x^{2}+y^{2}+z^{2}=0$ as double curve. The image surfaces of quadrics in $\mathbb{R}^{3}$ with respect to inversion $\mathbf{x}^{\prime}=\mathbf{x} /\|\mathbf{x}\|^{2}$ are typically Darboux cyclides. The inverse images of a cone or a cylinder of revolution or a torus is typically a Dupin cyclide.

Dupin cyclides are also quite popular in Computer Aided Geometric Design, in particular their applications for blending surfaces, see [13]. They are special instances of double Blutel conic surfaces [5], also known as supercyclides [1,14]. These surfaces carry two families of conics being contained in two pencils of planes where tangent planes along the conics form quadratic cones. The images of Dupin cyclides with respect to projective mappings are supercyclides. 
A Dupin cyclide is the envelope of two one-parameter families of spheres, whose centers are contained in a pair of confocal conics. Moreover, Dupin cyclides are rational surfaces having rational offset surfaces. Thus they admit rational parameterizations with rational unit normal vectors. This property holds for all rational canal surfaces (envelopes of one-parameter families of spheres) but typically it is difficult to characterize canal surfaces with rational lines of curvature. Rational offset surfaces with rational nets of planar lines of curvature have been investigated in detail in [12]. According to this contribution there exist two classes of rational surfaces with planar lines of curvature. Their construction is based on orthogonal families of circles in the unit sphere $S^{2}$. With regard to these networks of circles in $S^{2}$, the surfaces are given by rational solutions of particular second order partial differential equations.

Contribution: We present two families of rational canal surfaces generalizing Dupin cyclides with regard to their property of having rational lines of curvature. A surface $\Phi$ of the first family possesses a rational center curve $C$ on a rotational cylinder $G$ and its spheres touch a cross section plane of $G$. Dupin cyclides of order four are obtained for ellipses $C \subset G$ as center curves, thus $C$ is a planar section of $G$. The surfaces $\Phi$ of the second family generalize the Dupin cyclides of order three in a similar way. Their center curves are rational plane curves and their spheres touch a given plane which is perpendicular to the carrier plane of the center curve. All these rational canal surfaces $\Phi$ have rational offset surfaces, rational lines of curvature and rational principal curvatures. This implies that $\Phi$ has rational focal surfaces and rational Gaussian and mean curvature. Rational parameterizations of these surfaces $\Phi$ and the mentioned invariants are given explicitly.

\section{$1.1 \quad$ Geometric Preliminaries}

Let a surface $\Phi$ be given by the parameterization $\mathbf{f}(u, v)$, where $(u, v)$ are coordinates in $\mathbb{R}^{2}$. Denoting the partial derivatives by $\mathbf{f}_{u}(u, v)$ and $\mathbf{f}_{v}(u, v)$, a normal vector of $\mathbf{f}(u, v)$ is computed by $\mathbf{n}(u, v)=\mathbf{f}_{u}(u, v) \times \mathbf{f}_{v}(u, v)$. The first fundamental form of $\mathbf{f}(u, v)$ is based on the scalar products of its partial derivatives,

$$
\|d \mathbf{f}\|^{2}=\left\|\mathbf{f}_{u} d u+\mathbf{f}_{v} d v\right\|^{2}=\left\|\mathbf{f}_{u}\right\|^{2} d u^{2}+2 \mathbf{f}_{u} \cdot \mathbf{f}_{v} d u d v+\left\|\mathbf{f}_{v}\right\|^{2} d v^{2} .
$$

Using the abbreviations $E=\left\|\mathbf{f}_{u}\right\|^{2}, F=\mathbf{f}_{u} \cdot \mathbf{f}_{v}$, and $G=\left\|\mathbf{f}_{v}\right\|^{2}$, it is written as

$$
\begin{aligned}
\|d \mathbf{f}\|^{2}=E d u^{2}+2 F d u d v+G d v^{2} & =(d u, d v) \cdot\left(\begin{array}{cc}
E & F \\
F & G
\end{array}\right) \cdot(d u, d v)^{T} \\
& =(d u, d v) \cdot I(\mathbf{f}) \cdot(d u, d v)^{T}
\end{aligned}
$$

The right hand side of (2) defines a local metric on the surface $\Phi$ and serves to measure lengths and areas in $\Phi$.

Assuming $\mathbf{n}(u, v)$ to be a unit normal vector of $\mathbf{f}(u, v)$, the coefficients of the second fundamental form are $L=\mathbf{n} \cdot \mathbf{f}_{u u}, M=\mathbf{n} \cdot \mathbf{f}_{u v}$, and $N=\mathbf{n} \cdot \mathbf{f}_{v v}$. Since the 
identities $\mathbf{f}_{u} \cdot \mathbf{n}=\mathbf{f}_{v} \cdot \mathbf{n}=0$ hold, $L$ can also be expressed by $-\mathbf{f}_{u} \cdot \mathbf{n}_{u}$. Analogous expressions hold for $M$ and $N$ and we note that $\mathbf{n}_{u}$ and $\mathbf{n}_{v}$ are tangent vectors of $\Phi$. The second fundamental form of $\mathbf{f}(u, v)$ reads

$$
\begin{aligned}
L d u^{2}+2 M d u d v+N d v^{2} & =(d u, d v) \cdot\left(\begin{array}{cc}
L & M \\
M & N
\end{array}\right) \cdot(d u, d v)^{T} \\
& =(d u, d v) \cdot I I(\mathbf{f}) \cdot(d u, d v)^{T} .
\end{aligned}
$$

The principal directions of $\mathbf{f}(u, v)$ are eigenvectors of $I I(\mathbf{f})$ with respect to $I(\mathbf{f})$ and the principal curvatures $\kappa_{1}$ and $\kappa_{2}$ are the respective eigenvalues. Considering a general rational surface, these functions are typically not rational.

A geometric characterization of lines of curvature on a given surface is as follows: A curve $C$ on a surface $\Phi$ is a line of curvature if and only if the normals of $\Phi$ along $C$ form a developable ruled surface. Lines of curvature are also characterized as surface curves having a Darboux frame which is rotation-minimizing with respect to the tangent vector of the curve, see [2].

We consider a parameterization $\mathbf{f}(u, v)$ of $\Phi$ with respect to lines of curvature which means that the $u$-lines as well as the $v$-lines are lines of curvature, thus $\mathbf{f}_{u} \cdot \mathbf{f}_{v}=0$. Consider the developable ruled surface $\mathbf{f}\left(u, v^{\star}\right)+t \mathbf{n}\left(u, v^{\star}\right)$ formed by the normals along a $u$-line $\mathbf{f}\left(u, v^{\star}\right)$ with $v^{\star}=$ const., and assume $\|\mathbf{n}\|^{2}=$ 1. The last condition implies $\mathbf{n} \cdot \mathbf{n}_{u}=0$, and we have $\operatorname{det}\left(\mathbf{f}_{u}, \mathbf{n}, \mathbf{n}_{u}\right)=0$ and consequently $\mathbf{n}_{u} \cdot \mathbf{f}_{v}=0$. Analogous considerations for the normals along $v$-lines lead to $\mathbf{n}_{v} \cdot \mathbf{f}_{u}=0$. These properties imply that $\mathbf{n}(u, v)$ is an orthogonal net of curves in the unit sphere $S^{2}$. In case of rational offset surfaces with rational lines of curvature, $\mathbf{n}(u, v)$ is a rational orthogonal net of curves in $S^{2}$.

\section{Canal Surfaces with Cylindrical Center Curve}

Consider the cylinder of rotation $Z: x^{2}+y^{2}=1$. A rational curve $M$ on $Z$ is parameterized by

$$
\mathbf{m}(u)=\left(\frac{1-f(u)^{2}}{1+f(u)^{2}}, \frac{2 f(u)}{1+f(u)^{2}}, r(u)\right)
$$

with rational functions $f(u)$ and $r(u)$. The substitution $\lambda(u)=2 \arctan f(u)$ implies

$$
\cos \lambda(u)=\frac{1-f(u)^{2}}{1+f(u)^{2}}, \sin \lambda(u)=\frac{2 f(u)}{1+f(u)^{2}}, \text { and } \dot{\lambda}(u)=2 \frac{\dot{f}(u)}{1+f(u)^{2}} .
$$

Thus, any rational curve on $Z$ can be parameterized by

$$
\mathbf{m}(u)=(\cos \lambda(u), \sin \lambda(u), r(u)) .
$$

We show that the envelope $\Phi$ of the one-parameter family of spheres

$$
S(u):\|\mathbf{x}-\mathbf{m}(u)\|^{2}-r(u)^{2}=0
$$

is a rational offset surface with rational lines of curvature. The Dupin cyclides of order four will appear as surfaces $\Phi$ for planar center curves $M$, see Fig. 1(b). 


\subsection{Parameterization of the Surfaces}

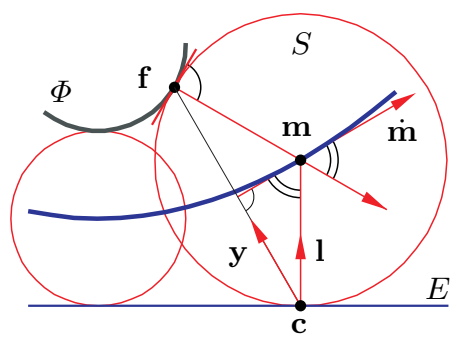

(a) Anticaustic mapping

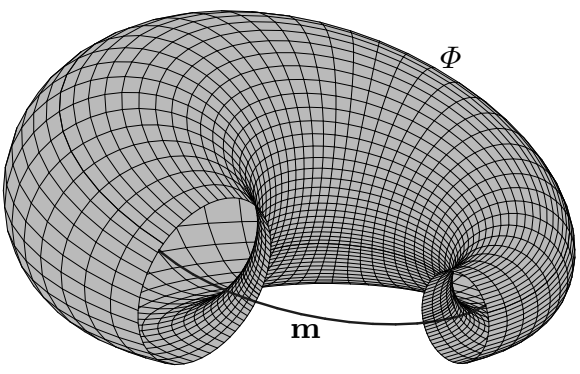

(b) Dupin cyclide of degree four

Fig. 1. Dupin cyclide and anticaustic construction

The spheres $S(u)$ touch the plane $E: z=0$ along the cross section curve $C: \mathbf{c}(u)=(\cos \lambda(u), \sin \lambda(u), 0)$. The characteristic circles $S(u) \cap \dot{S}(u)$ of $\Phi$ are the $u$-lines of the final parameterization $\mathbf{f}(u, v)$ and touch $E: z=0$ in points of C. $\Phi$ is constructed as anticaustic by reflection with respect to light rays parallel to $z$. To perform this construction, we reflect points $\mathbf{c}(u)$ of $C$ at the pencils of planes passing through the tangent lines of $M$, see Fig. 1(a). Here and in the following the derivatives of functions $x(u)$ are denoted by $\dot{x}$ whereas partial derivatives of bivariate functions $x(u, v)$ are denoted by $x_{u}$ and $x_{v}$. Derivatives of functions $x(v)$ are denoted by $x_{v}$.

Let $\dot{\mathbf{m}}(u)=(-\dot{\lambda} \sin \lambda, \dot{\lambda} \cos \lambda, \dot{r})(u)$ be a tangent vector of $\mathbf{m}(u)$. To perform the mentioned reflection one needs to parameterize the pencil of planes through the tangent line $\mathbf{m}+t \dot{\mathbf{m}}$. A normal vector of a plane of the pencil is a linear combination of two vectors orthogonal to $\dot{\mathbf{m}}$, for instance

$$
\begin{aligned}
& \mathbf{a}(u)=(-\cos \lambda,-\sin \lambda, 0)(u), \text { and } \\
& \mathbf{b}(u)=\dot{\mathbf{m}} \times \mathbf{a}=(\dot{r} \sin \lambda,-\dot{r} \cos \lambda, \dot{\lambda})(u) .
\end{aligned}
$$

Thus these normal vectors are parameterized by $\mathbf{y}(u, v)=\gamma(u, v) \mathbf{a}(u)+\mathbf{b}(u)$, with some function $\gamma(u, v)$ to be determined. The reflection of $\mathbf{c}(u)$ at planes through the tangent lines of $M$ is consequently given by

$$
\mathbf{f}(u, v)=\mathbf{c}+2 \frac{\mathbf{y} \cdot(\mathbf{m}-\mathbf{c})}{\|\mathbf{y}\|^{2}} \mathbf{y} .
$$

We still have to find a suitable function $\gamma(u, v)$ for the parameterization of $\mathbf{y}(u, v)$. The function $\mathbf{f}(u, v)$ is a parameterization with respect to lines of curvature if and only if the function $\gamma(u, v)$ satisfies

$$
\dot{r} \dot{\lambda}^{2}-\gamma_{u} \dot{\lambda}+\ddot{\lambda} \gamma=0
$$


One obtains the solution $\gamma(u, v)=\dot{\lambda}(u)(r(u)+g(v))$, with some function $g(v)$. Using the abbreviation $\alpha=\dot{r}^{2}+\dot{\lambda}^{2}\left(1+(g+r)^{2}\right)$, a representation of the canal surface $\Phi$ with respect to lines of curvature is

$$
\mathbf{f}(u, v)=\frac{1}{\alpha}\left(\begin{array}{c}
\left(\dot{r}^{2}+\dot{\lambda}^{2}\left(1+g^{2}-r^{2}\right)\right) \cos \lambda+2 r \dot{r} \dot{\lambda} \sin \lambda \\
\left(\dot{r}^{2}+\dot{\lambda}^{2}\left(1+g^{2}-r^{2}\right)\right) \sin \lambda-2 r \dot{r} \dot{\lambda} \cos \lambda \\
2 r \dot{\lambda}^{2}
\end{array}\right) .
$$

Consider rational functions $r(u), f(u)$ and $g(v)$. The substitution (5) implies that (10) is a rational parameterization of $\Phi$ with respect to its rational lines of curvature. The corresponding rational unit normal vector $\mathbf{n}(u, v)$ of $\mathbf{f}(u, v)$ satisfying $\mathbf{n}_{u} \cdot \mathbf{n}_{v}=0$ is explicitly given by

$$
\mathbf{n}(u, v)=\frac{1}{\alpha}\left(\begin{array}{c}
2 \dot{\lambda}(\dot{\lambda}(r+g) \cos \lambda-\dot{r} \sin \lambda) \\
2 \dot{\lambda}(\dot{\lambda}(r+g) \sin \lambda+\dot{r} \cos \lambda) \\
\left(\dot{r}^{2}+\dot{\lambda}^{2}\left(-1+(r+g)^{2}\right)\right.
\end{array}\right) .
$$

Corollary 1. Let $Z$ be a rotational cylinder and let $E$ be a plane perpendicular to the generating lines of $Z$. Consider a rational curve $M \subset Z$ and the family of spheres $S(u)$ centered at $M$ and touching $E$. Then the canal surface $\Phi$ enveloped by the spheres $S(u)$ is a rational offset surface with rational lines of curvature, explicitly represented by (10).

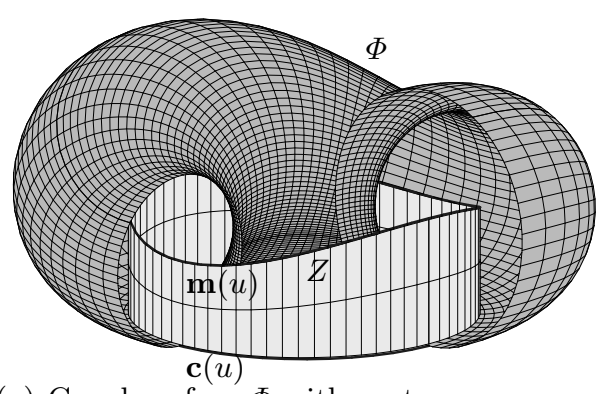

(a) Canal surface $\Phi$ with center curve $\mathbf{m}$ on a rotational cylinder $Z$

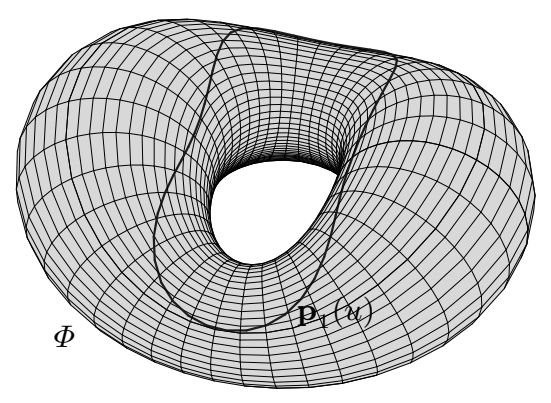

(b) Parabolic curve $\mathbf{p}_{1}$ on $\Phi$

Fig. 2. Canal surfaces with cylindrical center curve and parabolic line

Remark: The function $g(v)$ in $\mathbf{y}(u, v)=\dot{\lambda}(u)(r(u)+g(v)) \mathbf{a}(u)+\mathbf{b}(u)$ is responsible for the parameterization of the $v$-lines of $\mathbf{f}(u, v)$. We might set $g(v)=$ $v$. By this simple choice the parameterization $\mathbf{f}(u, v)$ misses the cross section $\mathbf{c}(u)=(\cos u, \sin u, 0)$. This can be corrected by replacing $g(v)$ by the quotient $g(v) / h(v)$. We omitted this here to keep the formulas as simple as possible, but we will return to this idea when computing the parabolic lines of $\Phi$ in equation (16). 


\subsection{Fundamental Forms and Curvatures}

Since $\mathbf{f}(u, v)$ is a parameterization with respect to lines of curvature, the first and second fundamental form are both represented by diagonal matrices. With the abbreviations $\alpha=\dot{r}^{2}+\dot{\lambda}^{2}\left(1+(r+g)^{2}\right)$ and $\beta=\dot{\lambda}^{3}\left(1+g^{2}-r^{2}\right)+\dot{\lambda} \dot{r}^{2}+2 r(\dot{r} \ddot{\lambda}-\ddot{r} \dot{\lambda})$, these matrices are

$$
\begin{aligned}
I(\mathbf{f}) & =\frac{1}{\alpha^{2}}\left(\begin{array}{cc}
\beta^{2} & 0 \\
0 & 4 \dot{\lambda}^{4} r^{2} g_{v}^{2}
\end{array}\right), \text { and } \\
I I(\mathbf{f}) & =\frac{1}{\alpha^{2}}\left(\begin{array}{cc}
2\left(\dot{r} \ddot{\lambda}-\ddot{r} \dot{\lambda}-\dot{\lambda}^{3}(r+g)\right) \beta & 0 \\
0 & 4 \dot{\lambda}^{4} r g_{v}^{2}
\end{array}\right) .
\end{aligned}
$$

The characteristic circles $S \cap \dot{S}$ of $\Phi$ are the $v$-lines of $\mathbf{f}(u, v)$ and are thus contained in the planes $\dot{S}:-x \dot{\lambda} \sin \lambda+y \dot{\lambda} \cos u+z \dot{r}=0$. These planes lie in a bundle with vertex $(0,0,0)$ and thus they envelope a rational cone. The eigenvalues of $I I$ with respect to $I$ are the principal curvatures

$$
\kappa_{1}=\frac{1}{r}, \kappa_{2}=\frac{2\left(\dot{r} \ddot{\lambda}-\ddot{r} \dot{\lambda}-\dot{\lambda}^{3}(r+g)\right)}{\beta} .
$$

It is clear that $\kappa_{1}$ does not depend on $v$ and is simply the reciprocal value of the radius $r(u)$ of the spheres $S(u)$, since one family of principal curvature centers is the center curve $\mathbf{m}(u)$ itself. In case of Dupin cyclides of order four, $\mathbf{m}(u)$ is an ellipse.

The product and the mean of the principal curvatures are known to be the Gaussian curvature and the mean curvature, and are given by

$$
\begin{aligned}
& K=\kappa_{1} \kappa_{2}=\frac{2\left(\dot{r} \ddot{\lambda}-\ddot{r} \dot{\lambda}-\dot{\lambda}^{3}(r+g)\right)}{r \beta}, \\
& H=\frac{\kappa_{1}+\kappa_{2}}{2}=\frac{\beta+2 r\left(\dot{r} \ddot{\lambda}-\ddot{r} \dot{\lambda}-\dot{\lambda}^{3}(r+g)\right)}{2 r \beta} .
\end{aligned}
$$

The set of principal curvature centers of a surface typically forms two surfaces, the focal surfaces $Q_{1}$ and $Q_{2}$. These focal surfaces are obtained by measuring the reciprocal values of $\kappa_{1}$ and $\kappa_{2}$ on the surface normals $\mathbf{f}+t \mathbf{n}$, with $\|\mathbf{n}\|=1$. In case of a canal surface $\Phi$, one focal surface becomes the center curve $\mathbf{m}(u)$. If both focal surfaces degenerate to curves, $\Phi$ is a Dupin cyclide, thus a canal surface in a twofold way. For canal surfaces $\Phi$, parameterized by (10) we thus obtain the center curve $\mathbf{q}_{1}(u)=\mathbf{f}(u, v)-r(u) \mathbf{n}(u, v)=(\cos \lambda, \sin \lambda, r)(u)$ as first component and a typically two-dimensional surface $Q_{2}$ as second component parameterized by

$$
\begin{aligned}
\mathbf{q}_{2}(u, v) & =\mathbf{f}(u, v)-\frac{1}{\kappa_{2}(u, v)} \mathbf{n}(u, v) \\
& =\frac{1}{\dot{r} \ddot{\lambda}-\ddot{r} \dot{\lambda}-\dot{\lambda}^{3}(g+r)}\left(\begin{array}{l}
(\dot{r} \ddot{\lambda}-\ddot{r} \dot{\lambda}) \cos \lambda-\dot{r} \dot{\lambda}^{2} \sin \lambda \\
(\dot{r} \ddot{\lambda}-\ddot{r} \dot{\lambda}) \cos \lambda+\dot{r} \dot{\lambda}^{2} \cos \lambda \\
\frac{1}{2}\left(\dot{\lambda}^{3}\left(-1+g^{2}-r^{2}\right)+\dot{\lambda} \dot{r}^{2}+2 r(\dot{r} \ddot{\lambda}-\ddot{r} \dot{\lambda})\right)
\end{array}\right) .
\end{aligned}
$$


A point on a surface is called parabolic if one of its principal curvatures vanishes. The parabolic points typically form the parabolic curves which separate regions with elliptic and hyperbolic surface points. Since $\kappa_{1}(u)$ only vanishes at the poles of $r(u)$ we investigate the parabolic lines corresponding to the zeros of $\kappa_{2}(u, v)$. For $g=\left(\dot{r} \ddot{\lambda}-\ddot{r} \dot{\lambda}-r \dot{\lambda}^{3}\right) / \dot{\lambda}^{3}$, and with the abbreviation $\delta=\dot{\lambda}^{4}\left(\dot{r}^{2}+\right.$ $\left.\dot{\lambda}^{2}\right)+(\ddot{r} \dot{\lambda}-\dot{r} \ddot{\lambda})^{2}-2 r \dot{\lambda}^{3}(\ddot{r} \dot{\lambda}-\dot{r} \ddot{\lambda})$ we obtain the parameterization

$$
\mathbf{p}_{1}(u)=\frac{1}{\dot{\lambda}^{4}\left(\dot{r}^{2}+\dot{\lambda}^{2}\right)+(\dot{r} \ddot{\lambda}-\ddot{r} \dot{\lambda})^{2}}\left(\begin{array}{c}
\delta \cos \lambda+2 r \dot{r} \dot{\lambda}^{5} \sin \lambda \\
\delta \sin \lambda-2 r \dot{r} \dot{\lambda}^{5} \cos \lambda \\
2 r \dot{\lambda}^{6}
\end{array}\right)
$$

of a parabolic line on $\Phi$, see Fig. 2(b). We obviously have lost the solution $\mathbf{p}_{2}(u)=\mathbf{c}(u)=(\cos u, \sin u, 0)$. This is corrected by replacing $g(v)$ by $g(v) / h(v)$ in $\kappa_{2}(u, v)$. Its numerator becomes $2 h\left(h(\dot{r} \ddot{\lambda}-\ddot{r} \dot{\lambda})-\dot{\lambda}^{3}(r h+g)\right)$ and the second parabolic line $\mathbf{c}(u)$ is obtained for $h=0$.

Theorem 1. Let $\Phi$ be a rational canal surface given by equation (10). $\Phi$ is a rational offset surface with rational lines of curvature and the principal curvatures as well as the focal surfaces are rational. These surfaces carry two parabolic curves, the cross section $C$ and the curve given by equation (16). The Dupin cyclides of order four are obtained for planar center curves.

\section{Canal Surfaces with Planar Center Curve}

As the last section generalizes Dupin cyclides of order four, we now deal with generalizations of Dupin cyclides of order three. These cyclides are canal surfaces in a twofold way as the previous ones, but their focal curves or center curves of the families of spheres are a pair of confocal parabolas.

Consider a rational curve $M$ in the plane $Z$. Without loss of generality we assume $Z: y=0$ and let $M$ be parameterized by

$$
\mathbf{m}(u)=(q(u), 0, r(u)),
$$

with rational functions $q(u)$ and $r(u)$. The construction of the family of surfaces generalizing the cubic Dupin cyclides follows similar lines as the construction presented in Section 2.

\subsection{Parameterization of the Surfaces}

These surfaces $\Phi$ are constructed as envelopes of a one-parameter family of spheres

$$
S(u):\|\mathbf{x}-\mathbf{m}(u)\|^{2}-r(u)^{2}=0,
$$

with center curve $M$ and radius function $r$. To construct $\Phi$ as anticaustic of reflection at $M$ with respect to light rays parallel to $z$, one needs to span the normal plane of $\dot{\mathbf{m}}$ by two independent vectors

$$
\begin{aligned}
& \mathbf{a}(u)=(0,1,0), \text { and } \\
& \mathbf{b}(u)=\dot{\mathbf{m}} \times \mathbf{a}=(\dot{r}, 0,-\dot{q}) .
\end{aligned}
$$


The general normal vector of the center curve is thus found by $\mathbf{y}(u, v)=\gamma(u, v) \mathbf{a}+$ $\mathbf{b}$, with a rational function $\gamma(u, v)$. To obtain a parameterization $\mathbf{f}(u, v)$ of $\Phi$, one performs a reflection of $\mathbf{c}(u)=(q(u), 0,0)$ at all planes passing through the tangents of $M$, which is realized by

$$
\mathbf{f}(u, v)=\mathbf{c}(u)+2 \frac{\mathbf{y} \cdot(\mathbf{m}-\mathbf{c})}{\|\mathbf{y}\|^{2}} \mathbf{y} .
$$

This parameterization is a representation of $\Phi$ with respect to lines of curvature if and only if $\gamma(u, v)=g(v) \dot{q}(u)$. The explicit parameterization reads

$$
\mathbf{f}(u, v)=\frac{1}{\dot{r}^{2}+\dot{q}^{2}\left(1+g^{2}\right)}\left(\begin{array}{c}
\left(\dot{r}^{2}+\dot{q}^{2}\left(1+g^{2}\right)\right) q-2 r \dot{r} \dot{q} \\
-2 r g \dot{q}^{2} \\
2 r \dot{q}^{2}
\end{array}\right),
$$

and it is not difficult to see that these surfaces have planar lines of curvature, where one family is contained in the planes $y+g z=0$. The second family are the characteristic circles $S(u) \cap \dot{S}(u)$ and these are contained in $\dot{S}(u): \dot{q} x+\dot{r} z=q \dot{q}$.

As in the previous section, $g(v)$ realizes the parameterization of the characteristic circles. Since we set $\mathbf{y}(u, v)=g(v) \dot{q}(u) \mathbf{a}(u)+\mathbf{b}(u)$ we miss the line $(q(u), 0,0)$ in $\mathbf{f}(u, v)$. This can be corrected by replacing $g(v)$ by a quotient $g(v) / h(v)$. We omit this here to keep formulas simple but apply it later for the computation of the parabolic line.

The unit normals of $\mathbf{f}(u, v)$ are

$$
\mathbf{n}(u, v)=\frac{1}{\dot{r}^{2}+\dot{q}^{2}\left(g^{2}+1\right)}\left(\begin{array}{c}
2 \dot{q} \dot{r} \\
2 g \dot{q}^{2} \\
\dot{r}^{2}+\dot{q}^{2}\left(g^{2}-1\right)
\end{array}\right)
$$

This parameterization of $S^{2}$ is also obtained by applying a stereographic projection $\sigma: \mathbb{R}^{2} \rightarrow S^{2}$ with center $(0,0,1)$ to the parameterization $(\dot{r} / \dot{q}, g)$ of $\mathbb{R}^{2}$. The normal vectors $\mathbf{n}(u, v)$ form an orthogonal net of circles in $S^{2}$, passing through the common point $(0,0,1)$ and having orthogonal tangents there. The following result is also contained in [12] which gives a full classification of rational offset surfaces with planar rational lines of curvature.

Corollary 2. Let $\Phi$ be a rational canal surface parameterized by (20), whose center curve $M$ is a plane rational curve $(q(u), 0, r(u))$ and whose spheres touch a line $\mathbf{c}(u)=(q(u), 0,0)$. Then $\Phi$ is a rational offset surface with rational planar lines of curvature.

\subsection{Fundamental Forms and Curvatures}

Analogously to Section 2, the first and second fundamental forms are both represented by diagonal matrices. Using the abbreviations $\alpha=\left(\dot{r}^{2}+\dot{q}\left(g^{2}+1\right)\right)^{2}$ and $\beta=\left(-\dot{q}^{3}\left(1+g^{2}\right)-\dot{q} \dot{r}^{2}+2 r(\dot{q} \ddot{r}-\ddot{q} \dot{r})\right.$, these matrices are 


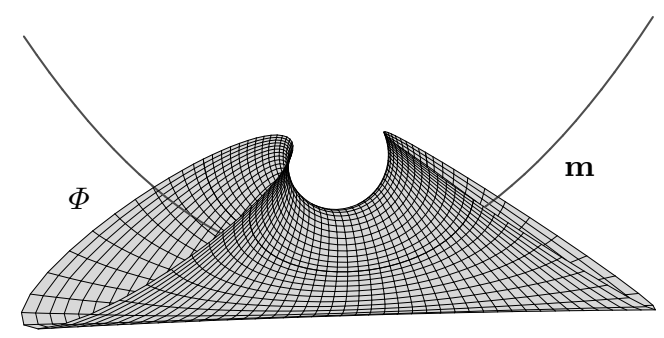

(a) Dupin cyclide of degree three

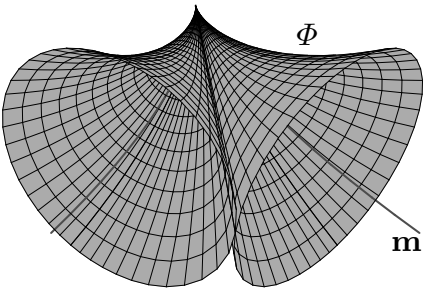

(b) Canal surface $\Phi$ with nodal cubic $\mathbf{m}$ as center curve

Fig. 3. Dupin cyclide of degree three and canal surface with planar center curve

$$
I(\mathbf{f})=\frac{1}{\alpha}\left(\begin{array}{cc}
\beta^{2} & 0 \\
0 & 4 r^{2} g_{v}^{2} \dot{q}^{4}
\end{array}\right), \text { and } I I(\mathbf{f})=\frac{1}{\alpha}\left(\begin{array}{cc}
2(\dot{q} \ddot{r}-\ddot{q} \dot{r}) \beta & 0 \\
0 & 4 r g_{v}^{2} \dot{q}^{4}
\end{array}\right) .
$$

The principal curvatures, the Gaussian and the mean curvature of $\Phi$ are

$$
\kappa_{1}=\frac{1}{r}, \kappa_{2}=\frac{2(\dot{q} \ddot{r}-\ddot{q} \dot{r})}{\beta}, K=\frac{2(\dot{q} \ddot{r}-\ddot{q} \dot{r})}{r \beta}, \text { and } H=\frac{\beta+2 r(\dot{q} \ddot{r}-\ddot{q} \dot{r})}{2 r \beta} .
$$

The set of focal points contains the curve $Q_{1}=M$ and the two-parametric surface $Q_{2}$ which is parameterized by

$$
\mathbf{q}_{2}(u, v)=\mathbf{f}-\frac{1}{\kappa_{2}} \mathbf{n}=\frac{1}{(\dot{q} \ddot{r}-\ddot{q} \dot{r})}\left(\begin{array}{l}
q(\dot{q} \ddot{r}-\ddot{q} \dot{r})-\dot{r} \dot{q}^{2} \\
-g \dot{q}^{3} \\
\frac{1}{2}\left(\dot{q}^{3}\left(1-g^{2}\right)-\dot{q} \dot{r}^{2}+2 r(\dot{q} \ddot{r}-\ddot{q} \dot{r})\right)
\end{array}\right) .
$$

Through the simple choice $g(v)$ for the parameterization of the characteristic circles we have lost the parabolic line $\mathbf{c}(u)=(q(u), 0,0)$. By replacing $g(v)$ by $g(v) / h(v)$ in $\kappa_{2}(u, v)$, its numerator becomes $-2 h^{2}(\dot{q} \ddot{r}-\ddot{q} \dot{r})$ and thus the parabolic line $\mathbf{c}(u)$ is obtained for $h=0$.

Theorem 2. Let $\Phi$ be a canal surface given by equation (20). $\Phi$ is a rational offset surface with rational lines of curvature and the principal curvatures as well as the focal surfaces are rational. The $x$-axis $\mathbf{c}(u)$ appears as parabolic line on $\Phi$. The Dupin cyclides of order three are obtained for parabolas with z-parallel axes as center curves $\mathbf{m}(u)$ in equation (17).

\section{Conclusion}

We studied particular rational canal surfaces with rational lines of curvature. These properties are invariant with respect to Möbius transformations, for instance the inversion $g: \mathbf{x}^{\prime}=\mathbf{x} /\|\mathbf{x}\|^{2}$. Möbius transformations are conformal and preserve spheres, where planes in $\mathbb{R}^{3}$ are also counting as spheres (with infinite 
radius). Applying $g$ to the first family of canal surfaces, one obtains canal surfaces $g(\Phi)$ with rational center curve $g(M)$ on a Dupin cyclide $g(Z)$, where the spheres $g(S)$ generating $g(\Phi)$ touch a fixed sphere $g(E)$ which is perpendicular to $g(Z)$.

Canal surfaces $\Phi$ of the second family are mapped under $g$ to canal surfaces $g(\Phi)$ with rational spherical center curve $g(M)$ since $g(Z)$ is a sphere. The spheres $g(S)$ defining $g(Z)$ touch a fixed sphere $g(E)$ being perpendicular to $g(Z)$.

\section{References}

1. Allen, S. and Dutta, D.: Supercyclides and blending, Comput. Aided Geom. Design 14, 637-651 (1997)

2. Biard, L., Farouki, R.T. and Szafran, N.: Construction of rational surface patches bounded by lines of curvature, Comput. Aided Geom. Design 27, 359-371, (2010)

3. do Carmo, M.: Differential Geometry of Curves and Surfaces, Prentice-Hall (1976)

4. Darboux, G.: Sur une classe remarquable de courbes et de surfaces algebrique, Gauthier-Villars, Paris, second ed., (1896).

5. Degen, W.L.F.: Nets with Plane Silhouettes. In: The Mathematics of Surfaces V, Design and Application of Curves and Surfaces, pp. 117-133. Oxford Univ. Press, (1994)

6. Dupin, C.: Applications de Geometrie et de Mechanique, Bachelier, Paris, (1822)

7. Krasauskas, R.: Branching blend of natural quadrics based on surfaces with rational offsets, Comput. Aided Geom. Design 25, May-June 2008, 332-341 (2008)

8. Krasauskas, R., and Mäurer, C.: Studying cyclides with Laguerre geometry, Comput. Aided Geom. Design 17, 101-126 (1999)

9. Peternell, M., Pottmann, H.: A Laguerre geometric approach to rational offsets, Comput. Aided Geom. Design 15, 223-249 (1998)

10. Pottmann, H. and Peternell, M.: Applications of Laguerre geometry in CAGD, Comput. Aided Geom. Design 15, 165-186 (1998)

11. Pottmann, H.: Rational curves and surfaces with rational offsets, Comput. Aided Geom. Design 12, 175-192 (1995)

12. Pottmann, H., Wagner, M.: Principal Surfaces, in: The Mathematics of Surfaces VII, Information Geometers Ltd., pp. 337-362 (1998).

13. Pratt, M.J.: Cyclides in computer aided geometric design, Comput. Aided Geom. Design 7, 221-242 (1990)

14. Pratt, M.J.: Quartic supercyclides I: Basic theory, Comput. Aided Geom. Design 14, 671-692 (1997)

15. Pratt, M.J.: Cyclides in computer aided geometric design II, Comput. Aided Geom. Design 12, 131-152 (1995)

16. Srinivas, Y.L., Kumar, V., Dutta, D.: Surface design using cyclide patches, Computer-Aided Design 28, 263-276 (1996). 